\title{
Endovascular Aneurysm Sealing for the Treatment of Ruptured Abdominal Aortic Aneurysms
}

Journal of Endovascular Therapy 2015, Vol. 22(3) 283-287 (c) The Author(s) 2015 (c) (1) \& Reprints and permissions: sagepub.com/journalsPermissions.nav DOI: $10.1177 / 1526602815582529$ www.jevt.org

\author{
Jorg L. de Bruin, MD', Jack R. W. Brownrigg, MRCS', \\ Alan Karthikesalingam, PhD, MSc, MA, MRCS', \\ Benjamin O. Patterson, PhD, MRCS', Peter J. E. Holt, PhD, FRCS', \\ Robert J. Hinchliffe, MD, FRCS', Robert A. Morgan, MRCP, FRCR', \\ Ian M. Loftus, MD, FRCS', and Matthew M. Thompson, MD, FRCS
}

\begin{abstract}
Purpose: To assess the feasibility and report preliminary results of ruptured abdominal aortic aneurysm (rAAA) repair with endovascular aneurysm sealing (EVAS), a novel therapeutic alternative whose feasibility has not been established in rAAAs due to the unknown effects of the rupture site on the ability to achieve sealing. Case Report: Between December 2013 and April 20I4, 5 patients (median age 7I years, range 57-90; 3 men) with rAAAs were treated with the Nellix EVAS system at a single institution. Median aneurysm diameter was $70 \mathrm{~mm}$ (range 67-91). Aneurysm morphology in 4 of the 5 patients was noncompliant with instructions for use (IFU) for both EVAS and standard stent-grafts; the remaining patient was outside the IFU for standard stent-grafts but treated with EVAS under standard IFU for the Nellix system. Median Hardman index was 2 (range 0-3). Two patients died of multiorgan failure after re-laparotomy and intraoperative cardiac arrest, respectively. Among survivors, all devices were patent with no signs of endoleak or failed aneurysm sac sealing at 6 months (median follow-up 9.2 months). Conclusion: EVAS for the management of infrarenal rAAAs appears feasible. The use of EVAS in emergency repairs may broaden the selection criteria of the current endovascular strategy to include patients with more complex aneurysm morphology.
\end{abstract}

\section{Keywords}

abdominal aortic aneurysm, ruptured aneurysm, endovascular aneurysm sealing, stent-graft, endobag, complications, mortality, aneurysm sac

\section{Introduction}

Although endovascular aneurysm repair (EVAR) of intact infrarenal abdominal aortic aneurysms (AAA) has been associated with a reduction in early mortality and morbidity as compared with open repair, the benefits of an endovascular strategy do not appear to translate to the treatment of ruptured aneurysms. Two randomized trials have demonstrated no difference in 30-day mortality when comparing open and endovascular repair of ruptured AAA. ${ }^{1,2}$

The Nellix endovascular aneurysm sealing (EVAS) system (Endologix, Irvine, CA, USA) offers a new approach to the endovascular treatment of AAA, achieving aneurysm exclusion by sealing the aneurysm sac. Each Nellix system consists of two identical catheter-based devices with a $10-\mathrm{mm}$ flow lumen being created by two balloon-expandable polytetrafluoroethylene-covered cobalt-chromium stents
(Figure 1). The stents are mounted on balloons for deployment and are surrounded by polyurethane endobags. While conventional endografts have a proximal and distal attachment zone for fixation, the EVAS device fixes the two stents within the aneurysm sac using the endobags, which are filled with a polyethylene glycol (PEG)-based hydrogel that conforms to the aneurysm flow lumen and solidifies within minutes of delivery, providing fixation and seal at the aortic neck and iliac arteries. Endobag filling is performed under

\footnotetext{
'St George's Vascular Institute, St George's University Hospitals NHS Foundation Trust, London, UK

Corresponding Author:

Jorg L. de Bruin, St George's Vascular Institute, St George's University Hospitals NHS Foundation Trust, 4th Floor St James Wing, Blackshaw Road, London, SWI7 0QT, UK. Email: jorgldebruin@gmail.com
} 


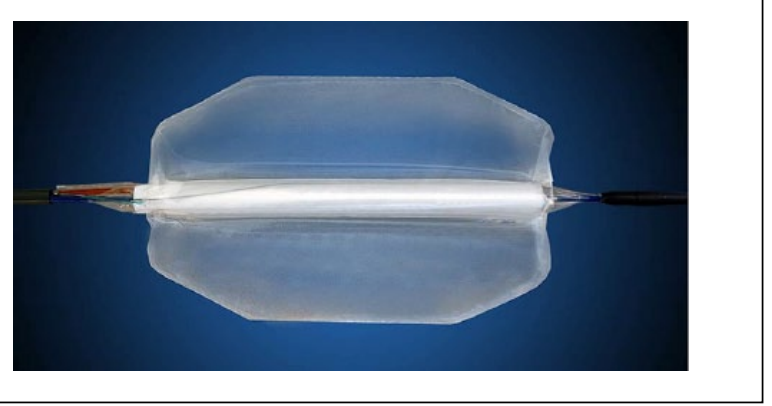

Figure I. Nellix endoprosthesis. Illustration courtesy of Endologix.

pressure monitoring, which allows the polymer to be instilled to a pressure of 180 to $220 \mathrm{~mm} \mathrm{Hg}$, which will seal the aneurysm. One of the potential difficulties in treating ruptured aneurysms with EVAS is whether the aneurysm rupture site will preclude the development of an adequate pressure during polymer fill to enable aneurysm sealing. Moreover, there is a theoretical risk of increasing the size of tear at the site of rupture.

Adverse aneurysm morphology remains a key challenge for conventional EVAR, and many devices are currently used outside the manufacturer's instructions for use (IFU), according to clinical need. The IFU accompanying the Nellix device suggest that an endovascular strategy may be feasible in a greater number of patients with a broader range of aortic morphology. ${ }^{3-5}$ We present our initial experience using EVAS in the treatment of patients with a ruptured infrarenal aneurysm.

\section{Case Series}

Prior to December 2013, 50 patients with AAA were treated with Nellix in an elective setting at our institute. All surgeons treating patients in the cases presented here had experience in $\geq 10$ elective cases using the Nellix device. Eligibility for EVAS in infrarenal ruptured AAA was established based on morphological suitability and the decision of clinicians with audited experience in emergency EVAR. Ruptured AAAs with morphology within the IFU for standard EVAR devices were not considered for EVAS.

Between December 2013 and March 2014, 5 patients (median age 71 years, range 57-90; 3 men) with ruptured infrarenal AAAs were treated with EVAS. All patients were conscious and lucid during the consent process and the novelty of this treatment option was explained to each before they provided written informed consent prior to aneurysm repair. Baseline characteristics, aneurysm morphology, and procedural details for each of the patients are displayed in Table 1. Aneurysm morphology in 4 of the 5 patients was noncompliant with the IFU for both Nellix and standard EVAR devices; the remaining patient was outside the IFU for standard EVAR devices but treated with Nellix under standard IFU for the Nellix system (Table 1).

Prospectively collected baseline clinical and morphology data included the Hardman index ${ }^{6,7}$ and aneurysm neck length, diameter, and angulation (Table 1). The decision to perform EVAS under local or general anesthesia was made according to clinical circumstance, with local anesthesia preferred if feasible. Centerline measurements on computed tomographic angiography (CTA) reconstructions were used to calculate aneurysm length and volume prior to surgery to allow stent sizing and thawing of the appropriate volume of polymer.

Local anesthesia was used in 4 cases, but 1 patient required general anesthesia (Table 1). Bilateral percutaneous access via the femoral arteries with ultrasound guidance was preferred, with Proglide closure devices (Abbott Vascular, Abbott Park, IL, USA) deployed at both access sites. However, a unilateral cutdown was required in 2 patients where percutaneous closure application failed.

After visualization of the lowest renal artery under angiographic control, a calibrated pigtail catheter was used to confirm the length to the bifurcation and subsequently the length of the devices needed. The median stent length was $160 \mathrm{~mm}$ (range 140-180) on the left side and $170 \mathrm{~mm}$ (range 140-180) on the right (Table 1).

The Nellix devices were inserted through the femoral access sites bilaterally and advanced over wires into position at the renal ostia. After retracting the covering sheaths, the stent-grafts were deployed by simultaneous inflation of the balloons within the stents to $7 \mathrm{~atm}$ (Figure 2A). The endobag was inflated first with a saline "pre-fill" to confirm the required polymer volume then with the aqueous PEG-based polymer (Figure 2B). Endobag fill pressures did not exceed $230 \mathrm{~mm} \mathrm{Hg}$. Five minutes following polymer curing, endobag pressures were checked again, and the delivery devices were removed. A secondary fill was performed in all patients. The stents were routinely postdilated using a 10- $\times 40-\mathrm{mm}$ angioplasty balloon (Cook Medical, Bloomington, IN, USA) inflated to $7 \mathrm{~atm}$. Completion angiography was used to demonstrate successful sealing of the ruptured aneurysm with bilaterally patent stent-grafts and iliac arteries. If the angle between the Nellix stent-graft and the iliac vessel wall was too large or ended at a curvature within the iliac artery, additional uncovered self-expanding stents (Cook Medical) were applied. In these 5 cases, a single adjunctive bare metal stent was deployed at the distal end of the EVAS device (iliac arteries) in 2 patients. A median of 3 units of blood was required during surgery, which lasted a median 85 minutes (interquartile range 75-90). Median intensive care unit stay was 3 days (range $0-6$ ) and median hospital stay was 8 days (range $0-40$ ) (Table 1 ).

Postoperative surveillance consisted of a predischarge duplex scan and CTA if postoperative renal function 
Table I. Baseline Characteristics, Aneurysm Morphology, and Procedural/ Postoperative Details.

\begin{tabular}{|c|c|c|c|c|c|}
\hline \multirow[b]{2}{*}{ Variables } & \multicolumn{5}{|c|}{ Patients } \\
\hline & $\mathrm{I}$ & 2 & 3 & 4 & 5 \\
\hline \multicolumn{6}{|l|}{ Baseline characteristics } \\
\hline Age, y & 90 & 57 & 70 & 85 & 70 \\
\hline Sex & Male & Female & Female & Male & Male \\
\hline Blood pressure, $\mathrm{mm} \mathrm{Hg}$ & $91 / 62$ & $99 / 65$ & $105 / 55$ & $80 / 55$ & $145 / 99$ \\
\hline Hemoglobin, g/dL & 10.7 & 9.7 & 7.6 & 13.9 & 11.2 \\
\hline Creatinine, $\mu \mathrm{M} / \mathrm{L}$ & 104 & 604 & 225 & 144 & 119 \\
\hline Acute myocardial ischemia on ECG & Yes & No & Yes & Yes & No \\
\hline Loss of consciousness & No & No & No & No & No \\
\hline Hardman index (0-5) & 2 & I & 3 & 2 & 0 \\
\hline \multicolumn{6}{|l|}{ Aneurysm morphology } \\
\hline Maximum aneurysm diameter, $\mathrm{mm}$ & 68 & 70 & 75 & 91 & 67 \\
\hline Neck length, mm & 31 & 154 & 24 & 19 & 22 \\
\hline Neck diameter, mm & 23 & 19 & 24 & 34 & 33 \\
\hline Infrarenal $(\beta)$ neck angulation, deg & 62 & 147 & 58 & 94 & 56 \\
\hline Suprarenal $(\alpha)$ neck angulation, deg & 76 & 145 & 49 & 67 & 53 \\
\hline Maximum flow lumen diameter, $\mathrm{mm}$ & 46 & 56 & 52 & 60 & 42 \\
\hline \multicolumn{6}{|l|}{ Procedural/postoperative details } \\
\hline Ineligibility for open repair & Comorbidity & $\begin{array}{l}\text { Previous abdominal } \\
\text { surgery and peritonitis }\end{array}$ & Comorbidity & Comorbidity & Comorbidity \\
\hline ASA grade & 5 & 4 & 5 & 5 & 5 \\
\hline Blood required during surgery, units & 3 & 3 & 2 & 6 & 0 \\
\hline Local anesthesia & Yes & Yes & Yes & No & Yes \\
\hline Procedure duration, min & 90 & 75 & 85 & 90 & 65 \\
\hline Nellix stent length left/right, mm & $160 / 160$ & $170 / 170$ & $140 / \mid 40$ & $180 / 180$ & $160 / 170$ \\
\hline Adjunctive stent-graft & Yes & No & No & Yes & No \\
\hline Polymer volume, $\mathrm{mL}$ & 120 & 135 & 120 & 200 & 100 \\
\hline Percutaneous groin access closure, $\mathrm{n}$ & I & 2 & 1 & 2 & 2 \\
\hline Intensive care unit stay, $d$ & 3 & 4 & 6 & 0 & I \\
\hline Hospital stay, d & 16 & 40 & 6 & 0 & 8 \\
\hline
\end{tabular}

Abbreviations: ASA, American Society of Anesthesiologists; ECG, electrocardiogram.

permitted; CTA was repeated at 6 months after discharge, and the duplex scan was done at both 3 and 6 months in an outpatient setting (Figure 3B). The surveillance requirements of EVAS remain to be defined, ${ }^{8,9}$ and this protocol differs from the duplex-only surveillance conducted following ruptured AAA repair with a conventional endograft.

Among the 5 patients treated with this device, 2 died. The first patient became hemodynamically unstable prior to surgery. Hemodynamic parameters deteriorated further following anesthetic induction; an endovascular sealing system was deployed, but the patient experienced cardiac arrest on the operating table and died. The other patient had hemodynamic instability on postoperative day 2; an emergency laparotomy was performed, and bleeding was observed from the aneurysm sac, assumed to result from a proximal type I endoleak. The device was explanted, and an aortobifemoral repair was performed. A colonic resection was subsequently performed for bowel ischemia on day 4 after
EVAS. The patient developed multiorgan failure and died 6 days after initial surgery.

Postoperative CTA performed in the 3 surviving patients demonstrated no endoleak and no limb occlusions. Median follow-up of surviving patients was 9.3 months; at 3 and 6 months, all 3 devices were patent on duplex ultrasound and CTA, with no evidence of endoleak or insufficient aneurysm sac sealing among the survivors.

\section{Discussion}

This report summarizes a preliminary clinical experience with the Nellix device for ruptured AAA repair. In contrast to EVAR devices, the Nellix system uses a sac-anchoring endoprosthesis for aneurysm exclusion. The small numbers in this report do not permit comparison with existing EVAR devices or open repair; however, they do support the feasibility of EVAS for ruptured infrarenal aneurysm repair in 


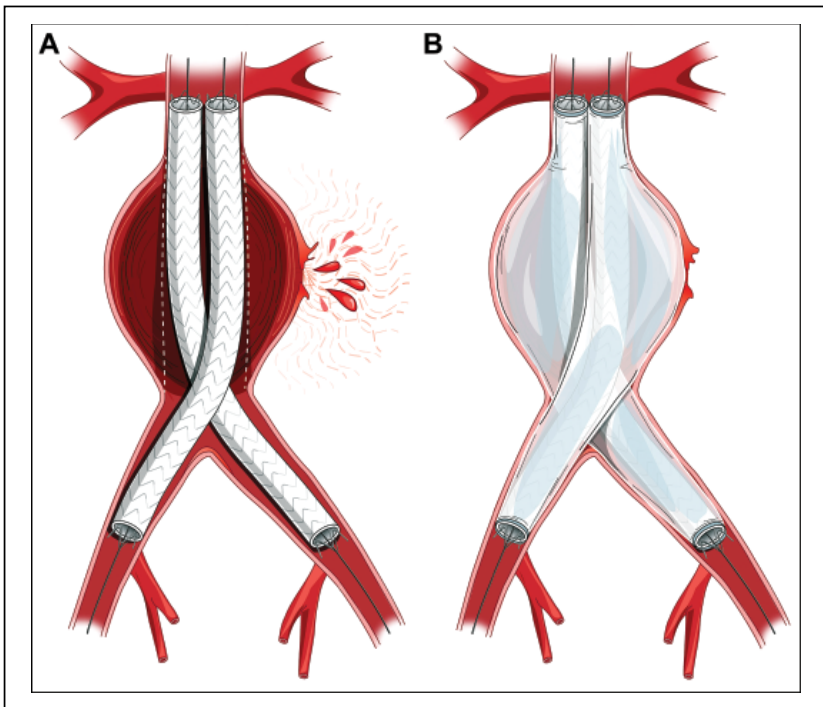

Figure 2. (A) The Nellix stents deployed within a ruptured infrarenal aneurysm, with the endobags not insufflated. (B) The Nellix device fully deployed with hemostatic control of the rupture and perfusion to both limbs.

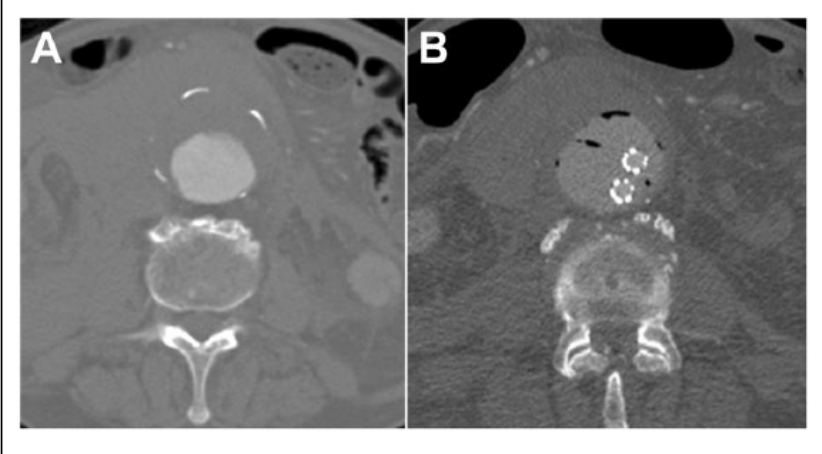

Figure 3. (A) Preoperative axial computed tomography angiography (CTA) scan of the aorta showing the hematoma surrounding the aneurysm, indicative of rupture. (B) The postoperative axial CTA with the endobags and stent-grafts within the aneurysm.

cases with morphological suitability, for example, those with a maximum aortic flow lumen diameter of $\leq 60 \mathrm{~mm}$. Nellix devices were successfully deployed without periprocedural events in 3 of 5 patients. EVAS may therefore provide an alternative to open or conventional endovascular repair of ruptured AAA.

The Nellix system offers several potential advantages over a conventional endografts for ruptured aneurysm repair. First, EVAS has been shown to be applicable to a wider range of aortic morphology (Figure 4) than currently available EVAR devices. ${ }^{3-5}$ The potential to use EVAS to treat patients who are noncompliant with the IFU of existing aortic stent-grafts is attractive in light of the higher

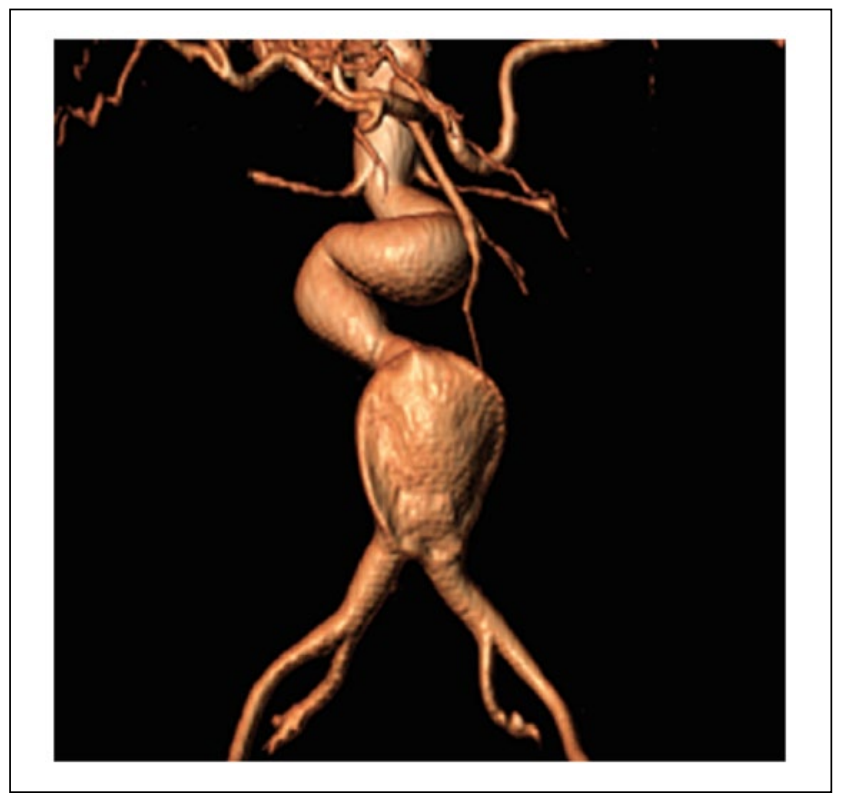

Figure 4. Preoperative computed tomographic angiography reconstruction showing hostile neck anatomy amenable to treatment with endovascular aneurysm sealing (EVAS).

incidence of sac expansion observed in patients treated by EVAR outside the IFU. ${ }^{10,11}$ The IFU for the Nellix devices specify that they are not suitable for patients with small or large common iliac arteries ( $<8$ or $>35 \mathrm{~mm}$, respectively) or those with a large patent flow lumen $(>60 \mathrm{~mm})$ within the aneurysm sac. ${ }^{3-5}$

The inventory of devices required for EVAS is less compared with that for conventional endografts, as the only sizing variation in the Nellix system is related to stent length. Preoperative sizing is therefore straightforward, involving the selection of only a suitable length of device, which may be of benefit in an emergency setting. ${ }^{2}$ The avoidance of contralateral limb cannulation in EVAS may reduce the overall procedure duration in certain cases. The Nellix system also provides an option for early hemostasis through endobag inflation with saline, while maintaining limb perfusion. A final advantage is the avoidance of type II endoleaks with aneurysm sac sealing.

The potential disadvantages of the EVAS technique for ruptured aneurysm repair include a risk of enlarging a rupture or tearing the aorta with the pressure used to fill the endobags. In addition, there is the potential for failure to seal a ruptured aneurysm due to an inability to generate the required pressure within the ruptured aneurysm sac. It is suspected that one patient in this cohort developed a type I endoleak that was not obvious on surveillance $\mathrm{CT}$ performed on postoperative day 1. Despite satisfactory appearances on the CTA, the patient was returned to surgery for laparotomy during a period of hemodynamic instability, where bleeding was observed 
from the aneurysm sac. As such, it would be reasonable to assume that a type I endoleak was responsible for reperfusion of the sac. On retrospective review of the postoperative images, there was opportunity to increase the proximal sealing zone by deploying the stent closer to the renal ostia. The detection of endoleaks with CTA in the early postoperative period after EVAS does pose a challenge due to the opacification of the polymer-filled endobags. The optimal imaging modality for surveillance at various postoperative periods remains to be determined.

Although preliminary, the current report of 5 patients provides the most substantial information to date about the feasibility of EVAS in ruptured aneurysm repair. The technique appears feasible, but clearly a refined protocol for the use of EVAS in ruptured AAA is required with consideration of the need for saline pre-fill and target polymer fill pressures in addition to identifying specific morphological parameters that predict success or failure.

In each patient there were several reasons for not selecting open repair, including previous laparotomy or peritonitis associated with continuous ambulatory peritoneal dialysis. In other cases, the patients were considered high risk for general anesthesia based on comorbidity, and a local anesthetic alternative was considered optimal. However, open repair should not be forgotten as a treatment option for ruptured AAA. An important consideration with any new device is durability, and longer follow-up is needed in a cohort of patients treated with EVAS to compare this modality with existing endovascular techniques.

\section{Conclusion}

Endovascular aneurysm sealing appears feasible for the management of ruptured aneurysms, with acceptable short-term outcome, albeit in a small series of patients. The application of EVAS devices for ruptured aneurysm repair may broaden the selection criteria of the current endovascular strategy to include patients with complex aneurysm morphology. ${ }^{13-15}$

\section{Declaration of Conflicting Interests}

The author(s) declared the following potential conflicts of interest with respect to the research, authorship, and/or publication of this article: Matthew M. Thompson provides consultancy to Endologix, Cook, and Medtronic.

\section{Funding}

The author(s) disclosed receipt of the following financial support for the research, authorship, and/or publication of this article: Matthew M. Thompson receives research funding from Endologix Inc. Peter J. E. Holt is a Clinician Scientist supported financially by the National Institute for Health Research (NIHR-CS-011-008), which had no role in the study design, data collection and analysis, decision to publish, or preparation of the manuscript.

\section{References}

1. Reimerink JJ, Hoornweg LL, Vahl AC, et al; Amsterdam Acute Aneurysm Trial Collaborators. Endovascular repair versus open repair of ruptured abdominal aortic aneurysms: a multicenter randomized controlled trial. Ann Surg. 2013;258:248-256.

2. IMPROVE Trial Investigators, Powell JT, Sweeting MJ, et al. Endovascular or open repair strategy for ruptured abdominal aortic aneurysm: 30 day outcomes from IMPROVE randomised trial. BMJ. 2014 Jan 13;348:f7661.

3. Donayre CE, Zarins CK, Krievins DK, et al. Initial clinical experience with a sac-anchoring endoprosthesis for aortic aneurysm repair. J Vasc Surg. 2011;53:574-582.

4. Krievins DK, Holden A, Savlovskis J, et al. EVAR using the Nellix Sac-anchoring endoprosthesis: treatment of favourable and adverse anatomy. Eur J Vasc Endovasc Surg. 2011;42:38-46.

5. Karthikesalingam A, Cobb RJ, Khoury A, et al. The morphological applicability of a novel endovascular aneurysm sealing (EVAS) system (Nellix) in patients with abdominal aortic aneurysms. Eur J Vasc Endovasc Surg. 2013;46: 440-445.

6. Hardman DT, Fisher CM, Patel MI, et al. Ruptured abdominal aortic aneurysms: who should be offered surgery? J Vasc Surg. 1996;23:123-129.

7. van Keulen JW, Moll FL, Tolenaar JL, et al. Validation of a new standardized method to measure proximal aneurysm neck angulation. J Vasc Surg. 2010;51:821-828.

8. Karthikesalingam A, de Bruin J, Patel SR, et al. Appearance of the Nellix Endovascular Aneurysm Sealing System on Computed Tomography: implications for postoperative imaging surveillance. J Endovasc Ther. 2015;22:297-302.

9. D'Abate F, Harrison SA, Karthikesalingam A, et al. Sonographic appearance following endovascular aneurysm repair using the Nellix endovascular sealing system. $J$ Endovasc Ther. 2015;22:182-186.

10. Schanzer A, Greenberg RK, Hevelone N, et al. Predictors of abdominal aortic aneurysm sac enlargement after endovascular repair. Circulation. 2011;123:2848-2855.

11. Karthikesalingam A, Nicoli TK, Holt PJ, et al. The fate of patients referred to a specialist vascular unit with large infrarenal abdominal aortic aneurysms over a two-year period. Eur J Vasc Endovasc Surg. 2011;42:295-301.

12. Jonker FH, Verhagen HJ, Mojibian H, et al. Aortic endograft sizing in trauma patients with hemodynamic instability. $J$ Vasc Surg. 2010;52:39-44.

13. Dijkstra ML, Lardenoye JW, van Oostayen JA, et al. Endovascular aneurysm sealing for juxtarenal aneurysm using the Nellix device and chimney covered stents. J Endovasc Ther. 2014;21:541-547.

14. Malkawi AH, de Bruin JL, Loftus IM, et al. Treatment of a juxtarenal aneurysm with the Nellix endovascular aneurysm sealing system and chimney stent. $J$ Endovasc Ther. 2014;21:538-540.

15. Niepoth WW, de Bruin JL, Lely RL, et al. In vitro feasibility of a sac-sealing endoprosthesis in a double chimney graft configuration for juxtarenal aneurysm. $J$ Endovasc Ther. 2014;21:529-537. 